This is an Author's Accepted Manuscript of an article forthcoming for publication in JOURNAL OF SCIENCE AND MEDICINE IN SPORT, copyright ELSEVIER:

https://www.journals.elsevier.com/journal-of-science-and-medicine-in-sport

ISSN: $1440-2440$

\title{
Medical care in unlicensed combat sports: A need for standardised regulatory frameworks
}

\author{
Alex Channon \\ University of Brighton, Hillbrow, Denton Road, Eastbourne, UK; School of Sport and Service \\ Management \\ Christopher R. Matthews \\ Nottingham Trent University, Clifton Campus, College Drive, Nottingham, UK; School of Science \& \\ Technology \\ Mathew Hillier \\ Leeds Beckett University, Cavendish 101 Headingley Campus, Leeds, UK; Carnegie School of Sport \\ Corresponding Author: \\ Dr. Alex Channon, School of Sport and Service Management, University of Brighton, Eastbourne, \\ UK \\ Tel: (+44)1273643746 \\ Email: a.channon@brighton.ac.uk
}




\section{Medical care in unlicensed combat sports: A need for standardised regulatory frameworks}

Alex Channon, Christopher R. Matthews and Mathew Hillier

\section{Abstract}

Objective: To explore the provision of medical care at 'unlicensed', full-contact amateur and lower-level professional combat sports competitions in England.

Design: Qualitative, mixed methods.

Methods: Observations totalling 200 hours of fieldwork shadowing medical professionals at 27 individual combat sports events, alongside formal, semi-structured interviews with 25 medical professionals, 7 referees and 9 promoters/event staff.

Results: Practices and standards vary widely. Event organisers and promoters often have very little understanding of how different types of medical practitioners operate. They rarely, if ever, check that the staff they are hiring are qualified, sometimes resulting in unqualified staff being used to provide medical cover at events. Venues are often poorly equipped to accommodate basic medical procedures. Patient confidentiality is very often compromised. Medical professionals often have limited autonomy within the combat sports milieu and may find themselves marginalised, with their judgements overruled by non-medical staff during competitive events. Some practitioners are cognisant of the dangers such working environments pose to their professional reputations and livelihoods, but remain working within combat sports regardless.

Conclusions: Despite pockets of good practice, the lack of standardised rules for medical care provision creates substantial risks to athletes, to practitioners and the standing of the profession. The development and implementation of standardised, enforceable regulatory frameworks for full-contact combat sports in England is urgently needed.

Keywords: Boxing; Martial Arts; Professionalism; Regulation; Sociological Factors 


\section{Introduction}

The provision of effective, high-quality medical care is a centrally important concern for sports authorities, with clear ethical and legal implications regarding the duty of care assumed by those who organise competitions. In this respect, governing bodies for sport typically produce policies for medical provision, specifying who is able to provide such support and how they ought to do so. However, sports events which take place outside of the remit of formal governing bodies, and are therefore not subject to any specific set of regulations, represent a troubling blind spot in the current regulatory environment.

Within the sport of boxing in England, formal governing bodies such as England Boxing and the British Boxing Board of Control provide regulation for amateur and professional fights respectively. As well as tracking fighters' medical histories within the sport, they also stipulate rules regarding the presence of ringside doctors and various procedures that should be adhered to. However, 'unlicensed' boxing events do not have to abide by governing body regulations, and in recent years, such events have become increasingly prominent throughout the UK; a case in point would be the rapid growth of 'white collar' amateur boxing shows'. Ultra White Collar Boxing - just one of several organisations in the country that promotes such unlicensed amateur fights - claims to have staged over 1000 events during the past 9 years ${ }^{2}$.

As with white collar boxing, mixed martial arts (MMA) and kickboxing shows are staged throughout the UK on most weekends of the year ${ }^{3,4}$. The lack of any formal governing body within the wider regulatory structures of UK Sport means little exists in the way of clear direction for the provision of medical care in these sports. One exception to this is the advisory body Safe MMA, who do offer such guidelines ${ }^{5}$ but lack powers to compel organisers of competitive events to adhere to them. As discussed below, it would appear that the majority of events below the highest level of competition do not voluntarily do so. Further, while numerous, self-regulating kickboxing associations exist, these do not have any clear 
accountability to wider sports regulators. They generally focus on setting rules and training referees and coaches, providing little advice regarding safety and medical care within competitions.

Past scholarship on the social organisation of sports medicine has raised various concerns about the maintenance of professional standards ${ }^{6}$, ethical (mis) $\operatorname{conduct}^{7,8}$, and risks to athletes arising through poor practices in this field ${ }^{9}$. In the present study we sought to investigate current practices within combat sports in England, specifically focusing on unlicensed events, hypothesising that a lack of enforceable regulation may provide a setting within which previously noted tendencies towards poor practice can thrive. Our research centred on the following question: without clear regulations guiding professional practice, how do medical practitioners work within the unique context of unlicensed combat sports?

\section{Methods}

This study adopted a qualitative design, employing a mixed-methods approach combining observations and semi-structured interviews. The observations were conducted by shadowing medical staff at combat sports events throughout England between 2017 and 2019 $(n=27)$, totalling 200 hours of fieldwork, and were recorded via field notes made throughout the course of each event. The majority of the events observed were MMA shows $(n=13)$, or mixed discipline shows $(n=5)$ that mostly featured MMA matches, but we also observed fight shows that featured only or mostly boxing $(n=5)$, kickboxing $(n=2)$, or Brazilian jiu jitsu $(n=2)$. With the exception of three internationally televised MMA shows, the majority of these events were classified within the field as being at the 'regional' level - that is, of a relatively low competitive standard with limited income and resourcing. Nevertheless, each of these shows featured intense, full-contact fighting, along with the various physical risks this entails ${ }^{10}$.

Interviews were conducted with medical professionals who had worked at such events $(n=25)$, as well as referees $(n=7)$ and show promoters/organisers $(n=9)$. Interviewees were 
purposively sampled, accessed through a combination of targeted invitations, chain-referral sampling and serendipitous contact in the field. Interviews lasted between 25 and 100 minutes and were digitally recorded for later transcription. Analyses of interview transcripts and observation field notes were completed using an iterative thematic analysis protocol.

Ethical approval for the study was granted by the first author's institutional review board; the ethical principles of the British Sociological Association were also adhered to throughout. Anonymity was maintained throughout the study by removing all potentially identifying information from notes and transcripts. Informed consent was received from all interviewees as well as the organisers of events and individuals shadowed during observations. Shadowing excluded the few instances where emergency treatment was provided during shows, as well as confidential interactions between medical staff and athletes.

\section{Results}

In combat sports, athletes come into contact with medical professionals almost exclusively at competitive events, with their presence organised and paid for by promoters. None of the medical staff in our sample had ever been asked about their qualifications or professional registration when being hired, nor were any of them formally interviewed. Rather, for all of the medical professionals in our study, personal recommendations shared within the combat sport community secured employment, reminiscent of informal hiring practices seen in other studies $^{11,12}$.

The amount a promoter was willing to spend on medical cover was usually key to deciding how many and what types of staff would be employed, and more specifically whether or not to have a doctor present. Among the medics we interviewed, it was commonly assumed that expensive medical cover was "the first corner to cut" when promoters tried to save money. As one paramedic pointed out, some "promoters (would) rather pay for ring girls, rather than pay for a doctor. They'll have five or six ring girls... but they won't pay for a doctor on the show." 
True to this, at several events we visited the ring girls outnumbered the medical staff, and were more of a consistent feature in the field than were doctors. Indeed, at most shows $(n=19)$, medical teams did not include doctors, being comprised of staff such as paramedics, nurses, emergency medical technicians (EMTs) and, in some cases, unqualified trainees or first aiders.

Aside from cost, promoters made decisions on who to hire based on informal criteria, and often had no conception of the roles that different practitioners were able to perform. One EMT told us they secured repeat work by presenting themselves at events as a thoroughlyprepared professional, partially indicated through the numerous large, bright red bags of kit they brought along. Although they never directly promoted themselves as anything other than an EMT, this self-presentation belied the fact that they were not insured to use all of this impressive equipment (which they told us they brought along so that if an external paramedic was called to an event and had forgotten something, they were prepared). The positive impression this gave off was confirmed in two separate interviews with promoters who had hired this particular medic, who explicitly referred to being impressed by the volume of kit they brought along.

Relying on positive impressions and word-of-mouth to secure employment failed to prevent certain other medical teams, referred to by some of our respondents as "cowboy outfits", from being hired. We shadowed one such team, who wore bottle green fatigues emblazoned with 'MEDIC' on the back despite only one of their members having formal medical qualifications or experience. This team's work was clinically and ethically questionable: two of the three were drinking alcohol before the show started and immediately after it ended; they failed to either notice or prevent the participation of two fighters with physical disabilities that put them at a clear competitive disadvantage against their able-bodied opponents; and they failed to perform a post-fight exam or offer follow-up treatment to a boxer who had been knocked out. While several others we interviewed explicitly identified this firm as prime examples of 
"cowboys", they continued to be hired to work on various shows around the country during the period of our study.

Pre-fight checks carried out by medical teams tended to be structured around taking physical observations such as heart rate, blood pressure and pupil dilation, along with a brief self-report measure of recent knockouts or injuries, current medications, allergies, and their family medical history. Only on the events which adhered to Safe MMA protocols did medics require fighters to be screened for blood-borne diseases or have brain scans. Interviews revealed that while some medics built their pre-fight procedures around governing bodies' guidelines used within licensed (boxing) events, most made them up based on procedures from their day jobs, or experience in the field.

None of our sample had been specifically trained in how to determine whether an athlete was fit to compete or not; several medics mentioned working with colleagues who did not understand how to interpret physical observations for this purpose. Furthermore, none of the medics in our sample had been trained for conducting such work in anticipation of contextspecific problems - such as the effects of fighters having 'cut weight' ${ }^{13}$, or their tendency to lie on pre-fight checks (which, several respondents told us, they do "all the time" to avoid being prevented from competing).

While conducting pre-fight consultations, confidentiality was often compromised ${ }^{6,14}$. At approximately two-thirds of the events we observed, fighters were interviewed about their medical history in crowded spaces - including, at three separate events, in the middle of publicly accessible corridors. This meant that coaches, event staff, and other fighters (including their opponents) were often present during the process. Checks were carried out in secure, private spaces within only three of the events we visited: the Safe MMA-compliant shows, each run by the same medical team. 
The presence of others during pre-fight consultations impacted on the disclosure of information between athletes and medics. At one show we observed a young teenage kickboxer's mother assuring the EMTs conducting pre-fight checks that her son's reported dizziness from a head injury earlier in the week was not serious, telling him that "you don't need to tell them about it - you talk too much!" The EMTs persisted in questioning the boy, who eventually agreed with his mother that he had overstated the injury, and he was subsequently cleared to fight.

When asked about the presence of others during consultations, some medics lamented that there was no other practical way to ensure all pre-fight checks could be done before show time, but others were more nonchalant. One paramedic even took it upon themselves to inform a boxer about her opponent's recent head injury in the hopes that she would "look after her in there" (rather than prevent the injured boxer from competing). When asked why, the paramedic responded that "there's no privacy here anyway... nothing's kept secret in this sport”.

Post-fight checks were a further example of wide disparity in practice. At the Safe MMAcompliant shows we observed, every fighter - win or lose - was routinely seen by a team of doctors in a backstage medical room after their fight. Elsewhere, medics performed a short series of physical checks on all fighters before they left the ring/cage. Some medics either did not do routine checks on all fighters, focusing only on those who'd lost or been knocked out, while still others did no post-fight checks at all. Twice we noted that medical teams did nothing in response to knockout or technical knockout stoppages, simply watching unconscious fighters from their cageside/ringside seats. Several interviewees told us similar stories of "useless", slow-to-act medical teams (including some doctors) they'd previously encountered.

Follow-up treatments and aftercare varied depending upon both the profession of the healthcare personnel present (e.g., EMTs were not able to suture open wounds, whereas 
nurses were) and the level of voluntary adherence to external protocols adopted at an event. Some MMA fighters (typically following head injuries) were issued temporary medical suspensions from all Safe MMA-compliant events by doctors who worked in accordance with its principles. However, this would not prevent them fighting on non-compliant MMA events or in other combat sports. Elsewhere, aftercare consisted of little more than "a quick phone call to the promoter" the day after a fight, while one respondent told us that they specifically avoided following up on fighters they treated: "I don't want to, we don't follow up. Because, you know, if they've died, I don't want to know about that".

Finally, we frequently noted that whatever procedures medics might opt to use, the lack of formal authority they often held in these settings could seriously impede their work. Although in some cases we witnessed a very high degree of cooperation and clear deference shown towards medics by event organisers, this was not guaranteed. Several medics reported uncooperative or downright obstructive treatment from others, such as coaches or promoters. This typically involved refusing them access to fighters to perform post-fight checks, or failing to withdraw fighters from competition after failing a pre-fight examination. Discussing two MMA fighters' pre-fight use of recreational drugs, one paramedic told us, "I signed them not fit to fight, but (the promoter) obviously had the capacity and the gumption to override that, so they fought anyway".

We witnessed such interference happening on several occasions, with one particularly striking example involving medical staff being ignored regarding a stoppage in a kickboxing match. Here, the promoter clearly and publicly overruled the medics in the middle of the ring, sending them back to their seats while they were attending to a hurt fighter, while asking the referee to continue the fight against their wishes - which he did. This was despite the fact that all referees and promoters we interviewed were unequivocal about never contesting a medic's advice to call off a fight; most medics (including those in question) assuring us they would not allow their professional opinion to be ignored in such circumstances; and several interviewees 
specifically describing the medic leading this team as a widely respected figure in the field whose judgement, in the words of one referee, "ought to be treated as gospel". With the unique vantage point afforded by our observational methodology, we were able to see very clear examples of how the idealisation of medical authority reported in most interviews could, at certain moments, be dismissed out of hand by those in positions of power in this field.

\section{Discussion and conclusion}

In this paper we have briefly outlined some of the findings from a study of the provision of medical care in unlicensed, full-contact combat sports events. Unlike the majority of their typical working environments, medical staff within such settings are provided little to no formal guidance on what they should be doing, resulting in a diverse range of practices. At best, these represent an attempt at replicating what staff do in the range of clinical settings they otherwise work in, which could be more-or-less suitable as a form of preparation to work within combat sports. At worst, medics' customised procedures may constitute both clinical and ethical failings, directly compromising the health of their patients. For instance, being questioned about factors impacting their fitness to compete in front of their peers and coaches/support teams - not to mention their opponents - adds to the well-documented social pressures on athletes to conceal information that could compromise their ability to participate in sport ${ }^{15-17}$, potentially leaving medics unaware of serious risks facing athletes in their care.

However, the lack of regulation which event organisers must adhere to greatly exacerbates such problems. Staff are hired on the basis of reputation, existing relationships and affordability rather than formal assessments of qualification, meaning that the only policing of standards depends upon the assessment of individuals who know next to nothing about clinical practice. Medics' roles at events are poorly defined and often misunderstood, which may bring them into unmanaged conflict with athletes as well as other players in the field, while they lack institutionally-mandated authority to deliver the care athletes ought to expect of them when such conflicts arise. 
Medical staff thereby risk being marginalised by those with greater power within the social milieu of a fight show, having their position reduced to a token presence. Not only are athletes thus placed at risk, but medical staff themselves may remain liable for what transpires against their judgement. With the lack of formal accountability and limited record-keeping at these unlicensed events, medical staff choosing to work in such environments are taking significant risks with their professional reputations and, as some interviewees reported, their livelihoods, should they be sued for negligence or malpractice in the event of serious harm befalling athletes in their care.

The development and implementation of standardised, enforceable regulatory frameworks for full-contact combat sports in England is therefore urgently needed. Although various guidelines do currently exist within relevant sports, and at times clearly have a positive impact, these are not legally mandatory, making them only partially effective in protecting athletes and practitioners. However, change may be forthcoming in the near future. The recent emergence of a new English Mixed Martial Arts Association, as well as the expanding ambitions of the Chartered Institute for the Management of Sport and Physical Activity, represent opportunities to better formalise the organisation of combat sports events in the country. Meanwhile, the continued work of Safe MMA, and the recent publication of a consensus statement on the management of concussions by the Association of Ringside Physicians ${ }^{18}$ are encouraging developments in terms of creating standardised guidelines for medics to work to. We argue that efforts to formalise the governance of all combat sports needs to accelerate, specifically with a view to enhancing the provision of medical care, in order to protect both the athletes and medical practitioners within them.

\section{Practical implications}


- Standardised, enforceable regulatory frameworks are needed to guide the provision of medical care within combat sports.

- Medical practitioners should ensure they establish clear parameters for their practice with relevant authority figures in the combat sports field, to protect their professional autonomy and promote athletes' welfare.

- Medical practitioners should be cognisant of the contextually-specific difficulties they may face when working within unregulated combat sports environments, and carefully weigh the risks that they take when engaging in this work.

\section{Acknowledgements}

This study was funded by an internal research grant from the University of Brighton. The authors wish to thank all of the participants who gave their time to this study, as well as the athletes and coaches who advised on the development of the research design, and colleagues who gave helpful comments on earlier drafts of this paper.

\section{References}

1. Wright E. Fast-track fisticuffs? An ethnographic exploration of time and white-collar boxing. Int Rev Sociol Sport Published Online First: 29 Nov 2018. doi:10.1177/1012690218815139.

2. Ultra White Collar Boxing. About UWCB. Available at: https://www.ultrawhitecollarboxing.co.uk/about/. Accessed 19 August 2019.

3. UK Fight Scene. Upcoming events. Available at: https://www.ukfightscene.co.uk/upcoming-events/. Accessed 19 August 2019.

4. Tapology. Mixed martial arts region: United Kingdom \& Ireland. Available at: https://www.tapology.com/regions/united-kingdom-ireland. Accessed 19 August 2019.

5. Safe MMA. Safe MMA: working for you and a safer mixed martial arts community. Available at: https://safemma.org/. Accessed 19 August 2019. 
6. Malcolm D. Sport, Medicine and Health: The Medicalization of Sport? London, Routledge, 2017.

7. Waddington I, Roderick M. Management of medical confidentiality in English professional football clubs: some ethical problems and issues. Br J Sports Med 2002; 36(2):118-123.

8. Devitt BM, McCarthy C. 'I am in blood Stepp'd in so far...': ethical dilemmas and the sports team doctor. Br J Sports Med 2010; 44(3):175-178.

9. Safai P. Healing the body in the 'culture of risk': examining the negotiation of treatment between sport medicine clinicians and injured athletes in Canadian intercollegiate sport. Sociol Sport J 2003; 20(2):127-146.

10. Jensen AR, Maciel RC, Petrigliano FA, et al. Injuries sustained by the mixed martial arts athlete. Sports Health 2017; 9(1):64-69.

11. Waddington I, Roderick M, Naik R. Methods of appointment and qualifications of club doctors and physiotherapists in English professional football: some problems and issues. Br J Sports Med 2001; 35(1):48-53.

12. Malcolm D, Scott-Bell A, Waddington I. The provision of medical care in English professional football: an update. J Sci Med Sport 2017; 20(12):1053-1056.

13. Hillier $M$, Sutton $L$, James $L$, et al. High prevalence and magnitude of rapid weight loss in mixed martial arts athletes. Int J Sport Nutr Exerc Metab Published Online First: 27 March 2019. doi:10.1123/ijsnem.2018-0393.

14. Malcolm D, Scott A. Practical responses to confidentiality dilemmas in elite sport medicine. Br J Sports Med 2014; 48(19):1410-1413.

15. Coker-Cranney A, Watson JC, Bernstein M, Voelker DK, Coakley J. How far is too far? Understanding identity and overconformity in collegiate wrestlers. Qual Res Sport Exerc Health 2018; 10(1):92-116.

16. Mayer J, Thiel A. Presenteeism in the elite sports workplace: the willingness to compete hurt among German elite handball and track and field athletes. Int Rev Sociol Sport 2018; 53(1):49-68. 
17. Walk S. Peers in pain: the experiences of student athletic trainers. Sociol Sport J 1997; 14(1):22-56.

18. Neidecker J, Sethi NK, Taylor R, et al. Concussion management in combat sports: consensus statement from the Association of Ringside Physicians. Br J Sports Med 2019; 53(6):328-333. 